\title{
EFFECT OF CORTISONE ON VASCULARIZATION AND OPACIFICATION OF THE CORNEA INDUCED BY ALLOXAN
}

\author{
$\mathrm{BY}$ \\ NORMAN ASHTON, CHARLES COOK, \\ AND MAURICE LANGHAM \\ From the Institute of Ophthalmology, London
}

THERE are few reports in the literature of experimental work dealing with the effect of cortisone upon corneal vascularization, and the evidence so far advanced is partly conflicting (Duke-Elder and Ashton, 1951), but there is already some indication from clinical observers that cortisone exerts a considerable inhibitory influence on the formation of new vessels in the diseased cornea. The experimental work reported in this paper investigates this subject further by the use of a recently evolved technique which has particular advantages for this study.

Before proceeding to investigate the effect of cortisone upon corneal vascularization it is necessary to consider the possible stimuli which might initiate new vessel formation; although the causes are still quite unknown, many theories have been advanced, some of which have an especial bearing upon the experimental conditions. Thus assuming for the moment that new vessel formation arises in response to a chemical substance liberated in traumatized tissue, as suggested by Michaelson and Campbell (1949), any effect of cortisone may be due to a neutralizing mechanism, and the extent of its action would then depend upon the size of the lesion and the quantity of cortisone administered. Similarly, the severity of the lesion would be of importance were vascularization to result from alterations in the normal tissue tension, as suggested by Cogan (1949). In attempting, therefore, to assess the effect of a given dose of cortisone upon corneal vascularization, it is essential that the experimental lesion should be, as far as variation in individual animals will allow, both standardized, in order to permit comparison, and the minimal required to produce vascularization, to ensure adequate sensitivity.

Although the work already published on this subject is of great importance in assessing the probable therapeutic value of cortisone, it cannot be held that caustic (Jones and Meyer, 1950; Leopold and others, 1951), or thermal burns (Lister and Greaves, 1951), are entirely satisfactory methods for investigating this problem, for the 
severity of such lesions may well either partially or completely obscure the cortisone effect.

Fortunately for our purpose one of us (Langham) has recently devised a technique for producing a minimal vascularizing lesion of the cornea which may be readily standardized. It was found that the injection of a solution of alloxan into the anterior chamber results in a marked swelling of the cornea accompanied by opacification of the stroma, which is followed, at approximately the sixth day, by a profuse ingrowth of blood vessels from the entire limbus. Preliminary experiments were carried out to discover the minimal dose of alloxan required to produce this effect and this quantity was used throughout the experiments.

\section{MethodS}

Experiments were carried out on adult rabbits weighing approximately $2.5 \mathrm{~kg}$. The thickness of the corneae was measured by means of the optical device described by Maurice and Giardini (1951). Pure alloxan monohydrate was dissolved in distilled water, neutralized, and made up to an isotonic concentration $(0.31 \mathrm{M}$.) immediately before administration. The cortisone used was the 11-dehydro-17-hydroxy corticosterone acetate (Cortone) of Merck and Co.

TeChNiQue of INDUCTION OF VASCUlarization BY Alloxan.-Measurement of the thickness of the corneae of all rabbits was made before beginning the experiments. Rabbits were rejected if there was a significant difference in the readings from the two eyes. Nembutal solution was used as a general anaesthetic. No application of a local anaesthetic was made, but if the animal proved to be too lightly anaesthetized, ethyl chloride was used to facilitate the injection of the alloxan solution into the anterior chamber. In order to avoid damage to the cornea, the injection was made through the sclera, the aqueous solution of alloxan being mixed with the aqueous humour by rapid movement of the plunger twelve times. In this manner the aqueous humour was replaced by a fluid containing a half isotonic concentration of alloxan. After the withdrawal of the needle, the anterior surface of the eye was washed with a warm isotonic saline solution. All subsequent readings of the thickness of the corneae, the degree of infiltration, and the extent of vascularization were made on the conscious animal.

EXPERIMENTS.-Two series of ten rabbits were used in the experiment. Five of the first series of ten animals were given subconjunctival injections of $7.5 \mathrm{mg}$. cortisone twice weekly. After $24 \mathrm{hrs}$ alloxan was injected into the anterior chamber of all ten rabbits.

In the second series, five of the animals were given daily intramuscular injections of $20 \mathrm{mg}$. cortisone, all ten rabbits again receiving an anterior-chamber injection of alloxan $24 \mathrm{hrs}$ after the initial intramuscular cortisone injection.

All the animals were thereafter examined daily, when the degree of corneal opacification and vascularization was noted and the corneal thickness estimated.

A diagrammatic representation of the appearances of the corneae of both control and cortisone-treated animals on these days is given in Figs 1 to 4 .

Graphs showing the relative changes in corneal opacity, density, and extent of vascularization (Figs 5, 6, and 7) illustrate the findings recorded in Tables I, II, and III.

Photographs were taken of all the affected corneae, on the 7 th, 11 th, and 17th days of the experiments. Figs 8,9 , and 10 show examples of the results obtained.

\section{RESULTS}

For simplicity the results will be considered under four main headings:

(1) Opacity of Cornea.-The degree of opacity of the cornea is recorded in Table $I$ and illustrated in Figs 1 to 5. 
Statistically, the degree of opacity of the cornea produced by the introduction of alloxan into the anterior chamber is much decreased in

ALLOXAN WITH SYSTEMIC CORTISONE
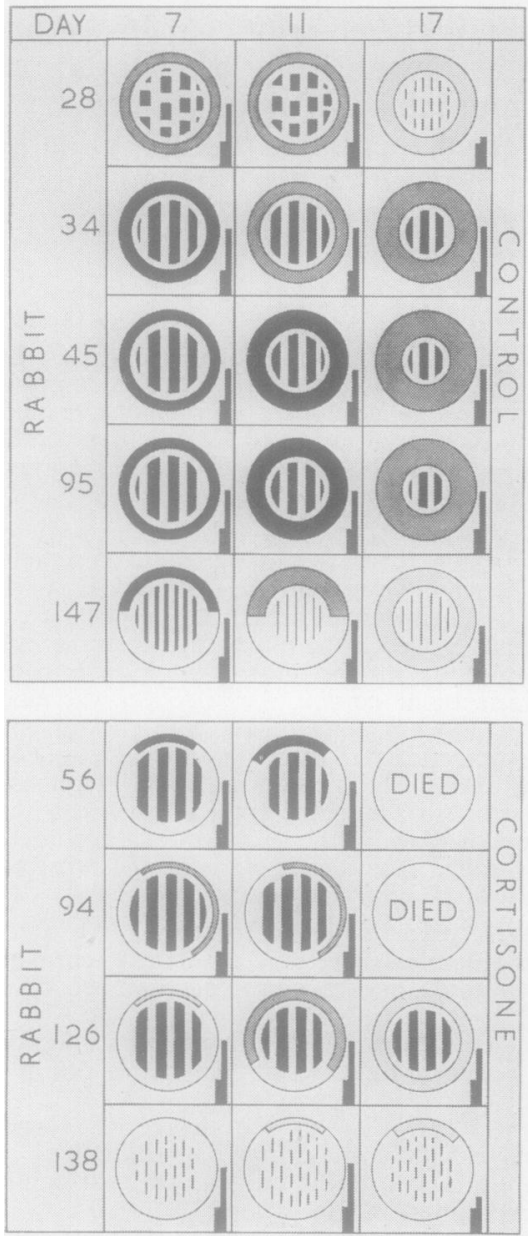

ALLOXAN WITH SUBCONJUNCTIVAL CORTISONE
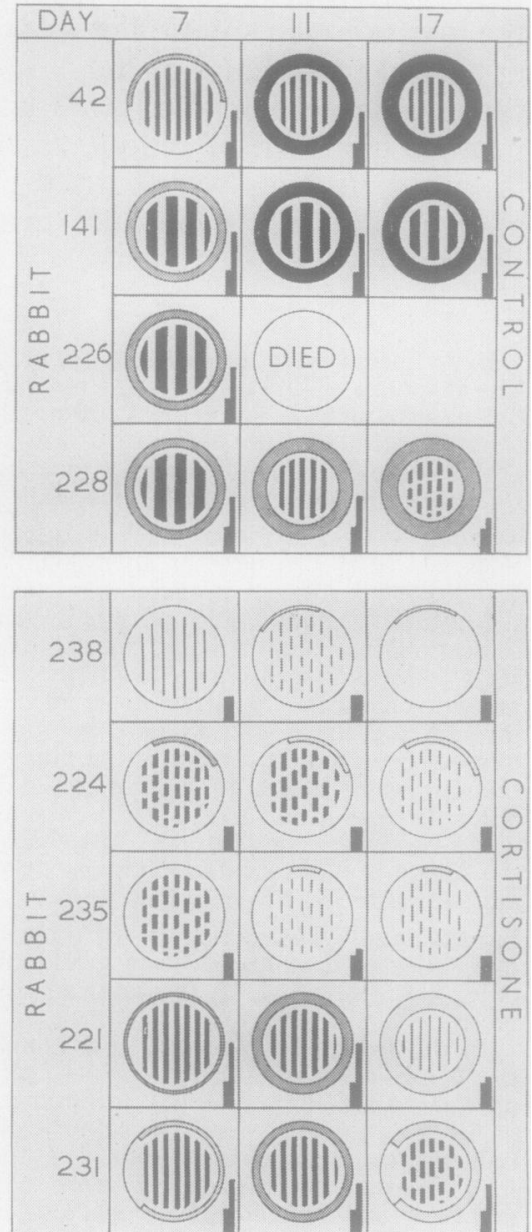

Figs 1 to 4.-Changes occurring after the injection of alloxan into the anterior chamber.

The greater circle represents the whole cornea. The extent of the ingrowth of blood vessels is shown by the size and width of the shaded ring. Density of vascularization is denoted by the tones of pale grey, grey, and black, which represent respectively the sparse, moderate, and dense ingrowth of blood vessels.

Three grades of opacity, i.e., slight, moderate, and dense, are shown by thin, medium, and thick continuous or discontinuous vertical lines. Where opacity is uniform over the whole cornea the vertical lines are continuous, where it is patchy the lines are broken.

The increase in thickness of the cornea is given by the height of the rectangular block, the thickness of the control cornea being given the value of unity in each case.

(One animal in each series died before vascularization began.) 
TABLE I

OPACITY OF CORNEA

\begin{tabular}{|c|c|c|c|c|}
\hline & \multirow{2}{*}{ Series } & \multicolumn{3}{|c|}{ Day of Experiment } \\
\hline & & 7 & 11 & 17 \\
\hline Controls ... & $\ldots$ & $5 \cdot 57 \pm 0 \cdot 31(7)$ & $5.0 \pm 0.57(7)$ & $4 \cdot 29 \pm 0 \cdot 84(7)$ \\
\hline \multirow{2}{*}{ Cortisone } & Subconjunctival & $3 \cdot 0 \pm 0.41(4)$ & $2 \cdot 25 \pm 0 \cdot 75(4)$ & $0.875 \pm 0.42(4)$ \\
\hline & Systemic & $4 \cdot 75 \pm 1 \cdot 24(4)$ & $4 \cdot 75 \pm 1 \cdot 24(4)$ & $3 \cdot 5 \pm 1 \cdot 88(2)$ \\
\hline
\end{tabular}

Results are expressed on an arbitrary range 0-6, where 0 represents the clear normal cornea and 6 the maximal degree of opacity observed.

All experimental results are expressed as the arithmetic mean and standard error. Number of experiments is expressed in brackets.

the group of animals receiving subconjunctival cortisone, whereas there is little difference between that shown by the animals treated systemically and the controls.

In all three groups there is a gradual clearing of the corneal opacity occurring after the 7 th day, but there is no significant difference in the relative rate of the decrease between the three groups of animals.

(2) Density of Vascularization.-It is apparent that the density of vascularization is much reduced in the animals treated with subconjunctival cortisone, but there is no significant difference statistically between that of the control group and that shown by the group of animals given

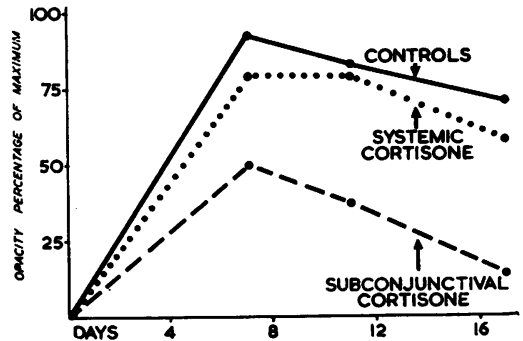

FIG. 5.-Opacity of cornea. systemic cortisone until after the 11 th day, when a significant reduction in density in the cortisone-treated animals becomes apparent (Table II; Figs 1 to 4 , and 6).

TABLE II

DENSITY OF VASCULARIZATION

\begin{tabular}{|c|c|c|c|c|c|c|}
\hline & \multirow{2}{*}{\multicolumn{3}{|c|}{ Series }} & \multicolumn{3}{|c|}{ Day of Experiment } \\
\hline & & & & 7 & 11 & 17 \\
\hline Controls ... & $\cdots$ & $\ldots$ & $\ldots$ & $2 \cdot 64 \pm 0 \cdot 18(7)$ & $2 \cdot 57 \pm 0 \cdot 17(7)$ & $2 \cdot 14 \pm 0 \cdot 26(7)$ \\
\hline \multirow{2}{*}{ Cortisone } & \multicolumn{3}{|c|}{ Subconjunctival } & $1.0 \pm 0.45(5)$ & $1 \cdot 40 \pm 0 \cdot 25(5)$ & $1.0 \pm 0.0$ \\
\hline & \multicolumn{3}{|c|}{ Systemic ... } & $1 \cdot 38 \pm 0 \cdot 72(4)$ & $1 \cdot 88 \pm 0 \cdot 49(4)$ & $1.0 \pm 0.0$ \\
\hline
\end{tabular}

Results are expressed on an arbitrary range 0-3, where 0 represents the clear normal cornea and 3 the maximal degree of vascularization observed.

All experimental results are expressed as the arithmetic mean and standard error. Number of experiments is expressed in brackets. 


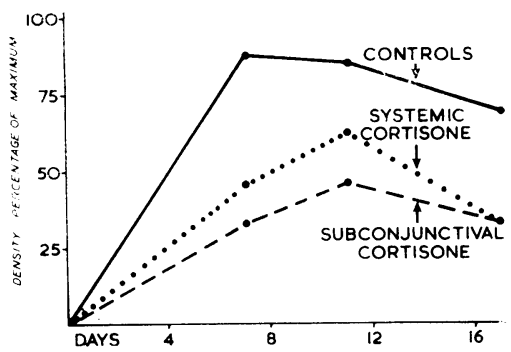

FIG. 6.-Density of vascularization.

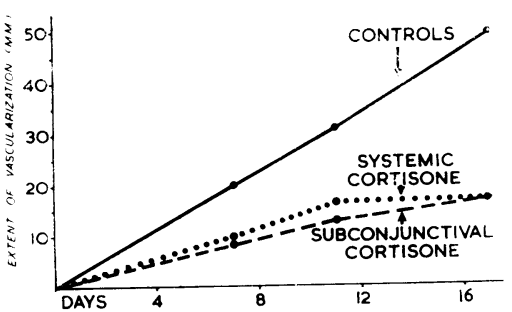

FIG. 7.-Extent of vascularization.

(3) Extent of Vascularization.-A marked decrease is shown by both groups of cortisone-treated animals throughout the experiment as compared with the control group, although in the case of the systemically treated animals this reduction was not evident in the very early stages of vascularization. It is noteworthy, however, that corneal vascularization was by no means entirely inhibited in either cortisone series (Table III; Figs 1 to 4 , and 7).

(4) Corneal Thickness.-After the introduction of alloxan into the anterior chamber, the thickness of the cornea rose rapidly in all series. Values

TABLE III

EXTENT OF VASCULARIZATION (IN MM. FROM LIMBUS)

Series

Day of Experiment

7

11

17

\begin{tabular}{|c|c|c|c|c|}
\hline Controls ... & $\ldots$ & $2 \cdot 0 \div 0.25(7)$ & $3 \cdot 1=0 \cdot 13(7)$ & $4 \cdot 94+0 \cdot 32(7)$ \\
\hline \multirow{2}{*}{ Cortisone } & Subconjunctival & $0.8 \pm 0.2$ & $1 \cdot 3 \pm 0 \cdot 33(5)$ & $1.7 \pm 0.49(5)$ \\
\hline & Systemic ... & $1.0=0.58$ & $1 \cdot 67=0.33$ (3) & $1 \cdot 67 \pm 1.5 \quad$ (2) \\
\hline
\end{tabular}

All experimental results are expressed as the arithmetic mean and standard error. Number of experiments is expressed in brackets.

TABLE IV

THICKNESS OF CORNEA

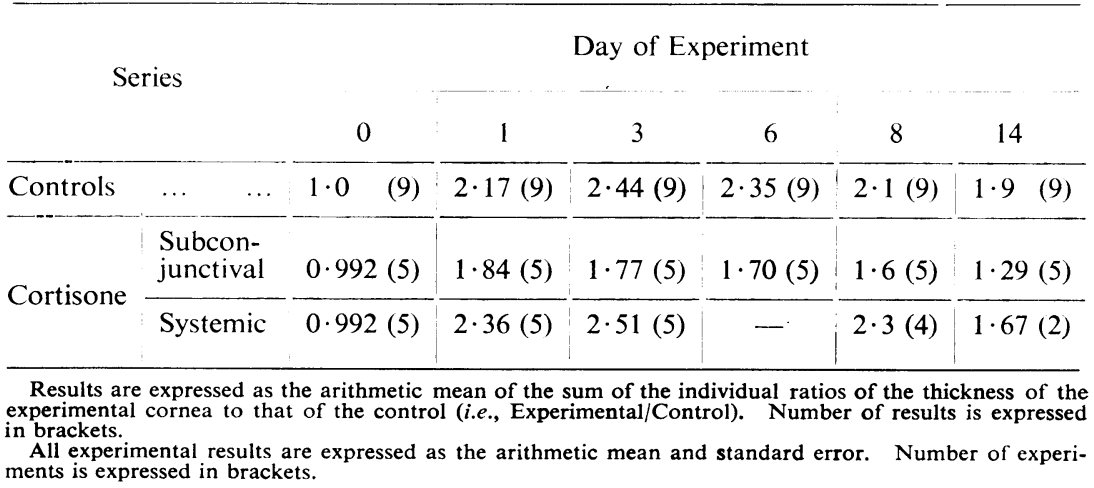


reached a maximum about the third day and then slowly began to fall. Corneae of animals treated with cortisone systemically showed no significant difference in thickness from those of the control series, whilst corneae of those treated by subconjunctival injection showed a significant difference from those of the controls, in that the increase in thickness was substantially less (Table IV; Figs 1 to 4 ).

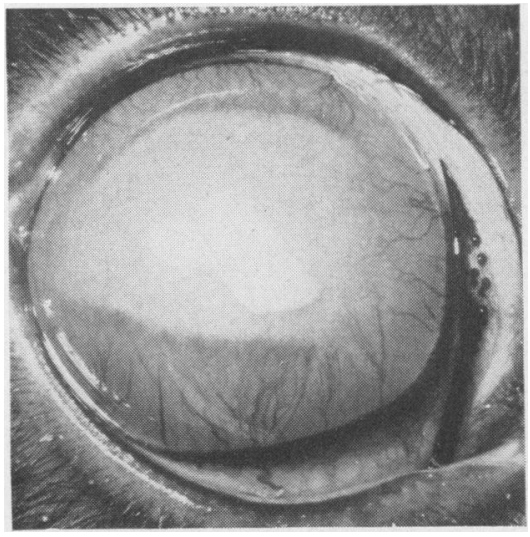

Fig. 8.-Control rabbit No. 141, 17th day. Note the marked opacity of the cornea and the dense ingrowth of new vessels advancing from the limbus on all sides.

\section{CONCLUSIONS}

The results obtained in these experiments (see Figs 8, 9, and 10) appear to demonstrate clearly that corneal vascularization, although not completely inhibited, is markedly reduced by the administration of cortisone. The time of onset of the inhibitory action varies, however, between those receiving subconjunctival injections and the systemically-treated group; a significant difference in the extent of vascularization in the systemically-treated animals was not obtained until after the 7 th day, and that in density of vascularization not until after the 11th day of the experiment. In

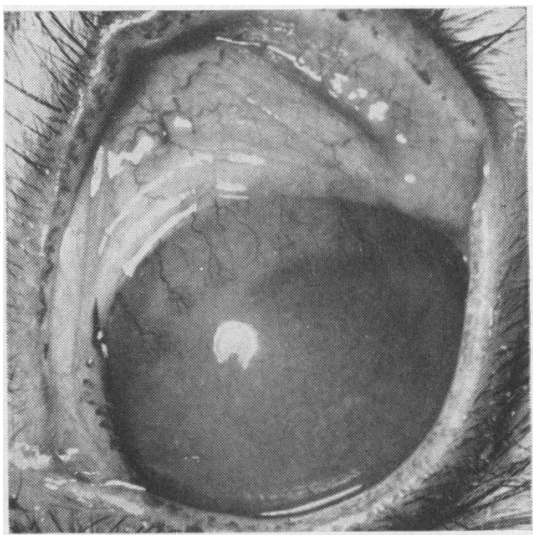

FIG. 9.-Systemic cortisone rabbit No. 126, 17 th day. Note that the corneal opacity is less marked than in the control animal (Fig. 8 ), and that the corneal vascularization, although equally advanced, is less dense.

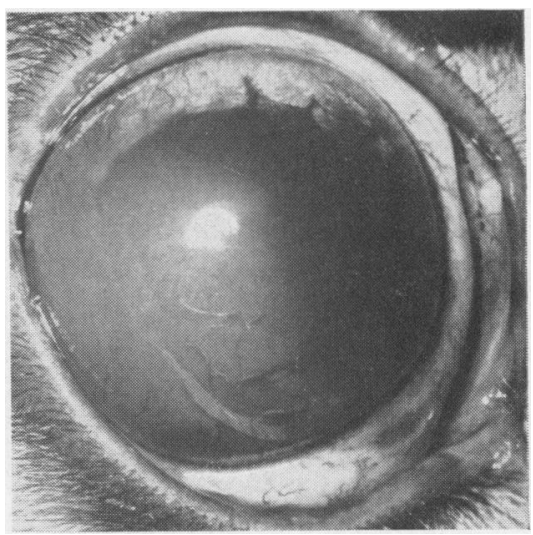

FIG. 10.-Subconjunctival cortisone rabbit No. 231, 17 th day. The corneal opacity is even less marked than in Fig. 9, and the corneal vascularization is patchy and sparse as compared with that in the control animal (Fig. 8). 
the animals given subconjunctival injections, on the other hand, the depression of vascularization both in density and extent was uniform throughout the experiment.

Systemic cortisone in these experiments appears to have had little or no significant inhibitory effect on the development of the corneal opacity, but when cortisone was given by the subconjunctival route a marked diminution of the corneal haze resulted. As was to be expected, the observations in corneal opacity were paralleled by those in corneal thickness; there was no significant difference between the thickness of the cornea in the control group and in the group treated with systemic cortisone, whereas those animals receiving subconjunctival cortisone showed a marked reduction in corneal thickness.

\section{SUMMARY}

(1) The effect of systemic and subconjunctival injections of cortisone on the vascularization, opacification, and swelling of the cornea induced by the intracameral injection of alloxan has been investigated in two series of ten rabbits.

(2) It has been shown that moderate doses of cortisone can markedly reduce, though not completely inhibit, corneal vascularization in the rabbit, and that this result is achieved more efficiently by subconjunctival than by systemic injection. Furthermore, a significant diminution in opacity and corneal swelling was obtained only when the cortisone was injected subconjunctivally.

We are indebted to the Medical Illustration Department, Institute of Ophthalmology, for the photographs and drawings. Our thanks are due to Miss E. FitzGerald and Mr. R. Dunbar for clerical and technical assistance.

The cortisone used in this investigation was supplied by the Medical Research Council and was in part derived from a gift from Merck and Co. made jointly to the Medical Research Council and the Nuffield Trust.

\section{REFERENCES}

Duke-Elder, S., and Ashton, N. (1951). British Journal of Ophthalmology, 35, 695. Camprell, F. W., and Michaelson, I. C. (1949). I Ibid., 33, 248.

Cogan, D. G. (1949). Arch. Ophthal., Chicago, 41, 406.

JONES, J. R., and MEYER, K. (1950). Proc. Soc. exp. Biol. N.Y., 74, 102.

Leopold, I. H., Purnell, E. J., Cannon, E. J., Steinmetz, C. G., and McDonald, P. R. (1951). Amer. J. Ophthal., 34, 361.

Lister, A., and GreAVES, D. P. (1951). British Journal of Ophthalmology, 35, 725. MaURice, D. M., and GiardinI, A. A. (1951). Ibid., 35, 169. 